\title{
La Personalidad del Psicoterapeuta y la Técnica Analítica ${ }^{1}$
}

\author{
Paula Campos ${ }^{2}$ \\ PsiRelacional, Lisboa
}

\begin{abstract}
En este texto, la autora parte de su experiencia como psicoterapeuta para pensar algunas cuestiones que se relacionan con la ubicua presencia de la personalidad del psicoterapeuta en su campo de trabajo. Es a partir de esa misma experiencia que discute la relación entre la personalidad del psicoterapeuta y la técnica analítica, o mejor, la práctica analítica, introduciendo conceptos e ideas de varios autores de la línea "relacional". Se relaciona la manera en que fue construida y empleada la técnica analítica con la necesidad de reducir el impacto de la subjetividad del analista y elige la relación psicoterapéutica entre dos sujetos que se dejan interpenetrar, como la relación transformadora por excelencia. Elige la intimidad del psicoterapeuta con su estructura e idioma personales como la mayor herramienta en el trabajo psicoterapéutico. Aboga por el desarrollo de un estilo analítico sintónico con el estilo integral del psicoterapeuta.
\end{abstract}

Palabras clave: personalidad del psicoterapeuta; la intimidad; técnica analítica vs. práctica analítica; psicoterapeuta en cuanto sujeto; estilo analítico

In this text, the author starts from her experience as a psychotherapist to think about some issues that relate to the ubiquitous presence of the personality of the psychotherapist in the field. It is from this same experience that he discusses the relationship between the personality of the psychotherapist and the analytic technique, or rather, the analytic practice, introducing concepts and ideas of several authors of the "relational" line. It relates how the analytical technique was constructed and employed with the need to reduce the impact of the analyst's subjectivity and elects the psychotherapeutic relationship between two subjects who allow themselves to be penetrated, such as the transforming relationship par excellence. It elects the psychotherapist's intimacy with his personal structure and language as the greatest tool in psychotherapeutic work. Advocates the development of an analytical style that is symptomatic of the integral style of the psychotherapist.

Key Words: personality of the psychotherapist; The intimacy; analytical technique vs. analytical practice; psychotherapist as subject; analytical style

English Title: Psychotherapist'Personality and Analytic Technique

\section{Cita bibliográfica / Reference citation:}

Campos, P. (2019). La personalidad del psicoterapeuta y la técnica analítica. Clínica e Investigación Relacional, 13 (2): 316-327. [ISSN 1988-2939] [Recuperado de www.ceir.info ] DOI: $10.21110 / 19882939.2019 .130202$

\footnotetext{
${ }^{1}$ Conferencia presentada en el Ciclo de Conferencias PsiRelacional en Lisboa el 19 de marzo el año 2019.

2 Psicóloga Clínica y Psicoterapeuta Psicoanalítica. Miembro fundador y de la Asamblea General de la PsiRelacional. Miembro de la IARPP.Correo electrónico: paulacampos99@hotmail.com CelR Vol. 13 (2) - Octubre 2019 ISSN 1988-2939 - www.ceir.info

(c) Derechos reservados/Copyright de Clínica e investigación Relacional y los autores. Prohibida la reproducción total o parcial sin autorización expresa. Este material es para uso científico y profesional exclusivamente y puede contener información clínica sensible. Los editores no se responsabilizan de los contenidos de los autores. Dirigir las consultas sobre derechos y autorizaciones a ceir@psicoterapiarelacional.es
} 
Soy una persona a quien le gustan las relaciones íntimas, es ese el terreno donde me siento bien. Soy una persona que tiene curiosidad por la forma en que el otro organiza la experiencia. Tengo un lado provocador, que le gusta sorprender al otro. Me gusta mucho cantar. Yo soy esto y muchas otras cosas.

¿De qué manera mis características influencian la forma en que soy psicoterapeuta?

Me parece evidente, por ejemplo, que el hecho de tener a varios pacientes que hacen psicoterapia con alta frecuencia (de sesiones) se relaciona con mi necesidad, capacidad y gusto por estar en relaciones donde suceda el compartir íntimo. ¿Cómo lo saben mis pacientes?

¿Y por qué hace unos años eso no sucedía, si ya estaba presente esa característica mía?

Me propuse a tratar el tema de la personalidad del analista y de su relación con la técnica y el camino que fui siguiendo en la construcción de esta conferencia, partió de mí, de mis cuestiones, de las cuestiones de los pacientes que conmigo se han encontrado y desencontrado, siendo, por tanto, un texto donde la teoría, mi personalidad y mi estilo personal se cruzan constantemente.

Recuerdo que estaba en el último año de mi formación como psicóloga y, en uno de los muchos cafés que bordean mi Facultad discutía con una colega la cuestión de la transformación de la personalidad. Yo abogaba:" el psicoanálisis es la única terapia que conduce a la transformación de la personalidad". Ella decía:"¿Cómo sabes?" Por supuesto, yo no tenía ninguna respuesta convincente más allá de la que mis maestros afirmaban, lo que para mí, en la época, era la verdad suprema. Quiero, con la evocación de este recuerdo poner en evidencia la cuestión del dogma analítico. El psicoanálisis fue pródigo en la construcción de dogmas: podemos alterar la personalidad de las personas, a través de la interpretación de sus conflictos inconscientes, interpretación que (ha de ser) realizada por un psicoanalista, en el contexto de la creación y mantenimiento de un setting riguroso, llamado setting analítico y mediante la utilización de la técnica analítica.

Yo diría que estamos ante el uso del dogma analítico para evitar enfrentar la plena congestión y el caos de la condición humana y de la tarea analítica.

La técnica analítica está, para mí, incluida en esta construcción dogmática, una vez que surge investida como un instrumento a través del cual podemos controlar todas las variables relevantes en el proceso analítico. El concepto de técnica incluye la idea de reglas sobre cómo proceder correcta y apropiadamente, cuyo propósito es, en parte, generar obediencia y reducir la influencia de la subjetividad individual (Coderch, 2014), subjetividad que en un primer momento fue patologizada, después controlada y hoy asumida como riqueza y elemento esencial en el proceso transformacional psicoterapéutico. 
La evolución de la teoría y de la práctica psicoanalítica fue ofreciendo diferentes visiones acerca del funcionamiento mental y consecuentemente del campo psicoterapéutico. Los autores que vinieron a poner el foco del desarrollo psíquico en la relación madre-bebé, como en la perspectiva de las relaciones de objeto, vinieron a transformar la clínica psicoanalítica en el sentido de una ampliación de la actitud clásica interpretativa de desvelamiento del inconsciente hacia una función relacional de proveer los elementos faltantes.

Y es de esta estrecha relación entre la teoría y la técnica que, a partir de Ferenczi, surge el analista auténtico, a partir de Bion el analista contenedor, a partir de Winnicott el analista sobreviviente, a partir de Kohut el analista empático, correspondiendo cada una de estas características a las características elegidas por estos psicoanalistas como las esenciales a tener y proveer el campo analítico. No creo que podamos adoptar la técnica de la empatía o de la autenticidad. Podemos, en el mejor de los casos, alcanzar una práctica con un buen equilibrio entre la autenticidad y su contrario o una práctica con un buen equilibrio entre la empatía y la falta de ella. Pero si esa práctica no deriva del mundo interno, de las vivencias y de las transformaciones del analista, o diciendo de otra forma, de su personalidad, entonces estaremos ante una "práctica técnica" y no ante una "práctica estilo". Por eso es que el concepto de técnica, para mí, es pernicioso, peligroso.

Por esos motivos, adoptaré, como Orange, Stolorow y Attwood (2012), en lugar del concepto de técnica, el concepto de práctica, que me parece más adecuado al espíritu psicoanalítico, al menos actual.

En el concepto de práctica se incluyen no sólo los principios relacionados con el linaje analítico del analista, sino también el estilo del analista, un proceso vivo que tiene su origen en la personalidad y la experiencia del mismo.

Para hablar de personalidad utilizaré la definición de Rodríguez Sutil (1998, 2005 cit. por Rodríguez Sutil, 2014) que entiende por personalidad el conjunto de las formas relativamente estables de sentir, pensar, comportarse y relacionarse con los demás.

En la tradición psicoanalítica, la personalidad del analista ha sido a menudo excluida de la comprensión del proceso terapéutico.

Parece que nos convencemos de que sería posible, a través de los análisis didácticos, liberarnos de todos los problemas, de modo que nuestra personalidad dejara de tener un papel preponderante en el campo psicoterapéutico. Como si el análisis didáctico nos pudiera transformar en una personalidad "limpia", en una personalidad modelo, a partir de la cual se producirian las interpretaciones correctas acerca de la realidad psíquica de las otras personas. Ahora bien, el análisis didáctico no sólo no nos transforma en individuos sin ansiedad ni nodos 
depresivos, como, naturalmente, no diluye nuestras características de personalidad, nuestro idioma personal, aquello que hace de nosotros ser quien somos. $Y$ es ese ser quien somos que entra en una comunicación consciente e inconsciente con los pacientes, contribuyendo en igual medida para la ocurrencia de los fenómenos psicoterapéuticos.

En la historia del psicoanálisis, la teoría de la contratransferencia fue la que permitió al psicoanalista hablar sobre su propia realidad emocional, procesos mentales y estados del Self. (Bollas, 1992)

Fue precursora de la posibilidad de que los analistas se representen como sujetos en el campo del análisis, posibilidad marcadamente revolucionaria de la concepción de campo y de relación analítica.

Antes de esa posibilidad vivimos tiempos psicoanalíticos marcados por la influencia de las teorías del desarrollo en las que la madre era descrita como objeto de las pulsiones del bebé y como objeto de satisfacción de las necesidades del bebé. Fue lento el reconocimiento de la madre como sujeto (Aron,1991), así como ha sido lento el reconocimiento del psicoterapeuta en cuanto sujeto.

El enfoque relacional se demarca por el intento de superar la dualidad sujeto-objeto (Coderch, 2014) y en esta lógica, el desafío para el analista, habituado a ser un objeto en la relación con sus pacientes de pasar a contar con la oportunidad aterradora y liberadora de ser un sujeto.

Pasa a formar parte de su tarea de descubrir una manera de ser un sujeto, una manera de expresar su estatus e idioma interior, utilizando el concepto de Cristopher Bollas.

Cuando me encuentro con el psicoanálisis relacional llevaba ya varios años de práctica clínica, de supervisión y de análisis personal. Llevó muchos años aprender la técnica psicoanalítica y aprender cuál o cuáles serían las mejores formas para ayudar a mis pacientes en sufrimiento, pero siempre en una lógica de que habría una técnica correcta, a pesar del estilo con que cada uno la pudiera poner en práctica.

Habituada a ocultar a mi Self para garantizar la neutralidad, la verdad es que no sabía cómo utilizar mi personalidad dentro del consultorio. No sabía bajo qué premisas utilizarla, no sabía si en la situación clínica la psicoterapeuta era más o menos importante que la persona, no sabía si lo que estaba más allá de la técnica era lícito (como por ejemplo, compartir mi opinión),

Las respuestas a estas preguntas fueron apareciendo e integrandose dentro de mí a medida que fui naciendo. Sí, naciendo. Bion (1987), en sus Seminarios Clínicos dijo: "no es posible hacer médicos o analistas-ellos necesitan nacer" (p.18) (como referido en Ogden, 2014). Pues así es, yo no sabía las respuestas a aquellas preguntas pues aún no había nacido como psicoterapeuta. Y en esta concepción que yo escogí, de que en los primeros años de mi práctica clínica yo 
todavía no había nacido como psicoterapeuta (correspondiendo esos años a una vida preanalítica), considero este nacimiento un nacimiento largo, difícil y muy laborioso.

Fue necesario descubrirme como persona y descubrir que la psicoterapeuta, vendría a nacer de cada objeto de mi propio Self a medida que me apropiara de mis objetos internos, integrándolos y vinculándolos a mis conocimientos y experiencias clínicas, elementos que vinieron a modificar mis objetos internos y a hacer nacer otros nuevos.

¿Cuáles son los orígenes de mis interpretaciones? ¿Por qué en un día el silencio de un paciente es agradable y en otro día insoportable? ¿Por qué selecciono un detalle de la narrativa y excluyo otros? Sólo podemos arriesgar respuestas a estas preguntas si incluimos en nuestro análisis, siempre, el análisis del mundo interior del analista. Mis interpretaciones y todas mis intervenciones son una mezcla del uso que el paciente hace de mí en la transferencia y de mi mundo interior. Gran parte del trabajo psicoterapéutico en el análisis ocurre dentro del psicoterapeuta, cuando éste procesa sus movimientos internos, como por ejemplo, su propia perturbación interior, a fin de dirigirse al paciente (Bollas, 1992).

Un elemento que me parece importante de considerar es la relación que el analista tiene con lo que no sabe y que no lo es. Mi no saber fue significado por mí de diferentes maneras en diversos momentos de mi recorrido profesional. Primero, sentía que mí no saber era responsable por el hecho de que todavía no era muy buena psicoterapeuta (no saber, como sinónimo de incapacidad); después, conforme acepté la inevitabilidad de las áreas de mi desconocimiento, sentía que esas mismas áreas no perjudicaban significativamente mi tarea psicoterapéutica (integración del saber y del no saber, no saber, como sinónimo de inevitabilidad) y en este momento, mí no saber, es representado por mí como un resultado de mi madurez y experiencia (no saber como sinónimo de realización y de espacio potencial). He encontrado en Cristopher Bollas una comprensión para este fenómeno y la caracterización de éste no saber como una realización, una especie de estructura mental necesaria para la creación de un espacio potencial, una pantalla analítica interior disponible para sentir y registrar el idioma del paciente (1992).

Lo que es curioso e interesante es que fue necesario mucho saber, estudio e inversión para alcanzar este lugar psíquico del no saber. Fue necesario saber, para comprender que el no saber no es una falla, sino un lugar. Un lugar de recepción, de interés, de curiosidad. Un lugar que me parece que se crea cuando saber y no saber se encuentran en una tensión equilibrada. Así que yo sé que sé, también sé que no sé, no sé que sé, y no sé que no sé. Cuando todas estas realidades pudieron coexistir dentro de mí, creo que fue cuando alcancé la tercera fase del proceso de desarrollo que he descrito anteriormente. Creo que un proceso similar ocurre en muchas áreas psíquicas. 
Me parece que lo que conduce a la intimidad con nosotros mismos es un proceso de apropiación de los objetos internos, que ocurre cuando pares de elementos que se oponen se encuentran en tensión: Amor-odio, generosidad-envidia, sensibilidad-insensibilidad, etc.

No puedo apropiarme, consistentemente, de mi capacidad de amar, si no me apropio consistentemente de mi capacidad de odiar. Este par, amor-odio, sólo puede ser integrado consistentemente cuando se encuentra en tensión, una tensión que, naturalmente, se pierde para recuperarse después. Creo que esta tensión es una especie de conflicto evolucionado, que naturalmente presupone la adquisición de la ambivalencia, pero que sólo se alcanza en un nivel maduro de la vivencia de la ambivalencia. La tensión es un tipo de conflicto irresoluble, es un punto óptimo en el que dos fuerzas que se oponen no pueden anhelar más que estar en tensión una con la otra. Si el conjunto formado por estos elementos en tensión es integrado en el Self tendremos una relación de intimidad con nosotros mismos. Es esa intimidad con nuestra estructura e idioma que me parece es la mayor herramienta en el trabajo psicoterapéutico. El más poderoso recurso práctico.

Ser íntima de mí misma significa que conozco muchas partes de mi Self y conozco las relaciones entre ellas. Significa, también, que las vivo de una forma más o menos directa, sin intermedio de muchas defensas psíquicas. Si un niño tiene la suerte de tener unos padres que le dejaron penetrar en sus mundos internos, siendo que tal movimiento es vivido por ambos como algo predominantemente bueno y al mismo tiempo, esos padres, tienen interés y curiosidad en el mundo interno del niño, entonces se lanzan las bases para una relación de intimidad del niño con ella misma. Y en este plano de la intimidad, hay como en todos los planes afectivos diferencias cuantitativas considerables. ¿Cuán lejos permitieron los padres que sus hijos penetrasen sus mundos internos? ¿Cuán lejos permitirá al analista que el paciente penetre su mundo interno? Diferencias cuantitativas y cualitativas producirán acontecimientos psíquicos muy diferentes.

\section{¿Cuál es la relación entre la intimidad y la salud mental?}

Estrecha. La intimidad se relaciona con generosidad y ésta con amor. La intimidad es antónimo de ansiedad. La intimidad es antónimo de retirada relacional. La intimidad se relaciona con la verdad. Pero, como decía antes, cualquier fenómeno psíquico sólo existe consistentemente si está en tensión con su opuesto. Una persona no puede ser verdaderamente generosa, si no conoce dentro de sí la envidia. En otras palabras, una persona no puede reconocer al otro como centro de experiencia equivalente al suyo, si no reconoce su propio Self y sus derechos a ser reconocidos por el otro, fenómeno que tan bien elucidó la psicoanalista Jessica Benjamin. 
La razón fundamental para que yo considere la relación de intimidad del analista con su propio Self la más poderosa herramienta práctica, se relaciona con el hecho de haber llegado a concluir que es la relación de intimidad entre analista y paciente un aspecto de vital importancia para el éxito de un análisis.

Aron (1991), a este respecto dice lo siguiente: "los esfuerzos del paciente para alcanzar al analista como persona representan la dimensión clave de la situación analítica, o sea el descubrimiento del analista en términos personales profundos es esencial en el proceso de curación o en lo que es curativo en el proceso proceso analítico. Ahora, pasamos de considerar la interpretación, después la dimensión transferencial-contratransferencial, y ahora la relación íntima profunda como las dimensiones clave del proceso psicoterapéutico.

Esta concepción provoca cambios técnicos inmensos, como por ejemplo pasar a entender la resistencia del paciente como un esfuerzo defensivo para lidiar con el analista y tal vez con la imposibilidad de descubrirlo profundamente (Wolstein, 1983 cit. por Aron,1991).

También las fantasías de los analizandos acerca de sus analistas pueden ser vistas como intentos para atrapar de manera única e idiosincrática, la ambigua y compleja realidad de su analista. No son sólo el resultado del pasado del paciente.

El resultado de estos cambios es que a medida que se analizan las resistencias y fantasías de los analizandos, los pacientes no sólo exponen más de su inconsciente pero también ganan conciencia de las áreas disociadas reprimidas de sus analistas (Aron,1991).

Cuando la psicoterapia es entendida como un proceso de interacción entre dos sujetos, todos los fenómenos producidos en el campo analítico son co-creados, por lo que comprender, por ejemplo, si fue la transferencia que precedió a la contratransferencia o viceversa se tornó menos importante que comprender cómo la experiencia conjunta de ambos sujetos nos ayuda a comprender mejor el analizando. No significa que las cuestiones de la práctica analítica más clásicas dejen de interesarnos, significa que en el aspecto relacional el enfoque de análisis es más amplio y más amplios también los factores de interés e importancia. Lo que me parece poco deseable es que tengamos una práctica enfocada en un aspecto principal, como, por ejemplo, la tendencia a traducir todo en interpretaciones transferenciales, o tener la tendencia a una adaptación constante al paciente. Lo que me parece deseable es que cada una de estas prácticas sea un elemento, entre muchos, dentro del campo analítico.

Por ejemplo, si la perspectiva del analista como sujeto se entiende de una forma distorsionada, podemos desembocar en una práctica que impone la subjetividad del analista. En la necesidad de establecerse como sujetos, los analistas pueden imponerse al paciente forzando a éste a 
asumir el papel de objeto. La imposición del analista de su propia subjetividad no es intersubjetividad.

Algo que fui descubriendo y construyendo es que la forma cada vez más frecuente de hacer interpretaciones es una forma en la que las interpretaciones no se anuncian como interpretaciones, que forman parte "de la conversación" en la que las ideas se formulan en lenguaje cotidiano. Lo que sucedió es que mi estilo analítico fue cada vez más parecido a mi estilo integral como persona, lo que a mí me parece algo muy deseable.

A veces, el estilo analítico y el estilo integral de una persona no están en sintonía y puede incluso suceder que determinado analista descubra posibilidades de ser más libre y auténtico en el ejercicio de su clínica, que en su vida en general y que ese sea un paso importante en la adquisición de la libertad y la flexibilidad en su personalidad. Pero, de cualquier forma, lo que me parece importante es que la función psicoterapéutica no sirva para los analistas sino en prácticas técnicas más o menos desligadas de su Self, para intentar conducir a los pacientes a ser lo que los analistas no lo son.

En la "práctica estilo", como en cualquier tipo de práctica, perdemos frecuentemente la capacidad de estar en intimidad con nuestros pacientes.

Cuando estoy momentáneamente menos disponible, para el paciente que está conmigo en sesión, me doy cuenta de que hago intervenciones más artificiales, que corresponden más al "lenguaje" analítico -como por ejemplo responder a una pregunta con otra pregunta-, o bien tener, en las características de mi voz, un tono menos involucrado, menos cerca de lo habitual, o por ejemplo preguntar "¿será?" en lugar de "¿piensa?". Diversos pacientes responden de diferentes maneras a este fenómeno, hay pacientes que se adaptan, hay pacientes que aceptan esta indisponibilidad temporal, hay pacientes que toleran y otros que no. Tengo un paciente que me imita con un tono jocoso cuando siente que mi tono es menos mío, que nació menos de mí, pero de una especie de mimetismo con un alter-ego analítico que pongo a funcionar mientras pueda estar concentrada en otra área de mi interior. "¿Será?", Dice el paciente con un aire de gozo. Me río, mostrando que estoy dispuesta a jugar con los objetos que los dos creamos allí.

El problema de la pérdida temporal de conexión íntima es algo natural, deseable, que promueve un equilibrio en la relación con el Self y con el otro. El problema de la no adquisición de una identidad psicoterapéutica desarrollada a partir del centro del Self y en su lugar de la aplicación de una técnica o la adopción de una actitud o postura genérica, es un problema que, según Mitchell $(1977,2015)$, invalida los análisis. 
Y, si es verdad que el más poderoso recurso práctico sea que el analista tenga una relación íntima consigo y desarrolle un estilo analítico sintonizado con su estilo integral, ¿será tan importante estudiar en profundidad la práctica y la teoría psicoanalíticas?

En mi opinión es muy importante estudiar en profundidad la teoría y la práctica analítica por dos razones principales:

1-Como dice Bollas (1992), los conceptos psicoanalíticos son objetos mentales cada uno con su propia función y su presencia constituye un potencial para un posible uso por el analizando. El hecho de incluir la personalidad del analista como un objeto de uso, no disminuye la necesidad del paciente poder contar con variados objetos, esenciales para las tres principales tareas analíticas: elaborar el Self verdadero a través de experiencias; tomar contacto con el inconsciente no pensado y articular el inconsciente reprimido. Cuantos más elementos el paciente pueda evocar, más posibilidades tendrá de expresar y desarrollar su idioma. Las teorías psicoanalíticas son elementos, la experiencia de vida del analista son elementos, las áreas del Self del analista disponibles para la relación, son elementos.

2-La presencia de una paradoja, necesito saber mucho acerca de la teoría y la técnica para olvidarme de ella. Necesito saber para no saber.

Haciendo una analogía entre dos áreas que me apasionan yo diría que, así como el músico estudia las notas, la estructura, la intención de la composición y las posibilidades interpretativas para poder olvidar todo eso durante el concierto en que él es la música, también el psicoanalista practica las teorías y actitudes psicoanalíticas para poder vaciarse de ellas en el consultorio: silenciar interiormente e intentar simple y naturalmente ser.

Volviendo a mí. Me encuentro con que, a pesar de que hay cierto tipo de personalidades con las que me es más fácil e inmediato el encuentro y la identificación, hay cada vez más, a medida que los años pasan, una variedad mayor de personas y estilos de personalidades con las que me encuentro afectivamente. Vuelvo al asunto de la intimidad. Si mantengo una relación conmigo más inclusiva, en la que vivo en relación integrada con diferentes partes de mi Self, eso me permite más fácilmente identificarse con los otros diferentes, pero en cierta medida, iguales a mí.

\section{Continuando en el tema de las compatibilidades ...}

En los diversos manuales de inspiración psicoanalítica acerca de la personalidad, del diagnóstico de la personalidad y de la comprensión de los meandros de funcionamiento en las diversas organizaciones de personalidad, a menudo se aconsejan técnicas específicas para manejar diferentes tipos de personalidad. No encontré una única referencia a la personalidad 
del analista, como elemento que entra en relación y modifica de forma única la expresión de la personalidad del paciente. En cuanto a mí, en lugar de trabajos titulados "Terapia Psicoanalítica con Pacientes Borderline" sería mucho más interesante habituarnos a ver trabajos como "Terapia Psicoanalítica con Pacientes y Analistas Borderline" o "Terapia Psicoanalítica con Terapeuta de Personalidad Fóbica y Paciente de Personalidad obsesiva ". Esto, si nos situamos en esta lógica más diagnóstica, porque evidentemente, aunque todos compartimos elementos de la personalidad, esos elementos y categorías universales no definen al sujeto humano.

Me gustaría resaltar cuatro dimensiones (Ogden, 2014) que pueden servir de guía para alcanzar una práctica "estilo", una práctica donde seamos uno de dos sujetos en el campo analítico.

1- Encontrar la forma de usar las cualidades singulares de la personalidad, así como desarrollar la capacidad de hablar de ellas.

2 - Atribuir riqueza y usar la experiencia propia como analista, analizando, progenitor, hijo, cónyuge, profesor, alumno, amigo, etc.

3- Desarrollar la capacidad de pensar de un modo que se base en, pero independiente, de la teoría y técnica analíticas de su analista, supervisor, colegas y antecesores analistas; el analista debe aprender teoría y técnica analítica de forma tan minuciosa hasta el punto de que un día pueda olvidarse de ellas.

4- La responsabilidad del analista inventar un nuevo psicoanálisis con cada paciente.

\section{Y para terminar, voy a hablar sobre el humor.}

Utilizo el humor fuera y dentro del consultorio. Tal vez porque, como dice María Rita Kehl (2005), el humor nos evita la fatiga de vivir.

El humor es una forma sublimada de reaccionar ante los dolores de la existencia: un modelo que piensa las contradicciones humanas con gracia. "Qué alivio tiene este artificio que hace, al menos por un instante, más leve la carga de enfrentarse a los reveses de la vida, el peso de calcular nuestros pasos, de reflexionar sobre nuestras elecciones e intentar predecir las consecuencias de ellas" (Kehl, 2005, P.53).

En 1927 Freud se refiere al humor como un don precioso y raro y una de las principales novedades que Freud trae en su trabajo es la idea de un Super-Ego bondadoso y tolerante.

La relación entre el Super-ego y el Ego que es generalmente crítica, exigente y severa, en el humor es lo opuesto. En ese caso el Super-Ego es capaz de jugar, como diciendo que el mundo fue hecho para gozar y no para un sufrimiento interminable. Tchekov escribió que la realidad 
se presenta en dos ventanas: una trágica y la otra cómica. El Super Ego también puede mostrar al Ego otra forma de mirar, una visión más ligera (Slavutzky, 2005).

Podemos, como todavía lo he hecho, usar la metapsicología para entender el humor, siempre y cuando eso no nos quite espacio para el buen humor, para reír, para simplemente ser y estar.

Y termino con una historia divertida. Voy a contar la historia que Abram Slavutsky (2005), un psicoanalista brasileño, contó a su analista en un día que quedó marcado como "el día en que su analista se rió". La historia fue ésta: en un congreso mundial de psiquiatría en Caracas, había algunos miles de colegas, y dos psiquiatras estadounidenses prepararon una investigación sobre la sexualidad humana. Demostraron que cuanto más frecuentemente era el sexo, más felices eran las personas. Al final de la presentación fueron aplaudidos y resolvieron lanzar un desafío. Pidieron que levantaran la mano a los que hacían sexo todos los días. Unos pocos, y surgieron risitas; después se les decía una vez por semana, una vez al mes y pocos se levantaban siempre avergonzados. Los presentadores pidieron, para concluir, que levantara la mano quien tenía sólo una relación por año. Un viejito, fumando pipa, tranquilo, levanta la mano de forma serena. Todos quedaron boquiabiertos y preguntaron al viejo psiquiatra si él podría explicar cómo un hombre que mantenía sólo una relación sexual por año podía estar tan feliz y tranquilo. Él sólo dijo: "Es hoy, es hoy, es hoy"!

\section{REFERENCIAS}

Aron, L. (1991).The Patients Experience of the Analyst Subjectivity. In Relational Psychoanalysis: the Emergence of a Tradition. New York: Taylor e Francis Group.

Bollas, (1992). Forças do Destino: psicanálise e Idioma Humano. Rio de Janeiro. Imago Ed.

Coderch (2014).Avances en Psicoanáisis Relacional: Nuevos campos de exploración para el psicoanálisis. Madrid: Ágora Relacional Editores.

Freud (1927).O Humor. Edição Standard Brasileira das Obras Psicológicas Completas de Sigmund Freud. Vol.XX. Rio de Janeiro:Imago Editora.

Kehl, M.R.(2005). Humor na Infância. In Slavutsky e Kuperman (organ.), Seria Trágico se não fosse Cómico: Humor e Psicanálise (p.51). Rio de Janeiro: Civilização Brasileira.

Orange, Stolororow e Attwood (2012). Trabajando Intersubjectivamente.Contextualismo en la práctica psicoanalítica. Madrid: Ágora Relacional Editores.

Mitchell, S. (1997,2015). Influencia y autonomia en psicoanálisis. Madrid: Ágora Relacional Ogden,T.(2014). Leituras Criativas: Ensaios sobre obras analíticas seminais. São Paulo: Escuta.

Ogden, T.(2004). This art os Psychoanalysis: dreaming undreamt dreams and interrupted cries. International Journal of Psychoanalysis, n.85, p.857-877. 
Rodriguez Sutil, C. (2014). Psicopatología psicoanalítica relacional: la persona en relación y sus problemas.Madrid: Ágora Relacional.

Slavutsky, A.(2005). O Precioso dom do humor. In Slavutsky e Kuperman (organ.), Seria Trágico se não fosse Cómico: Humor e Psicanálise (p.201). Rio de Janeiro: Civilização Brasileira.

Original recibido con fecha: 17/7/2019 Revisado: 15/09/2019 Aceptado: 30/09/2019 